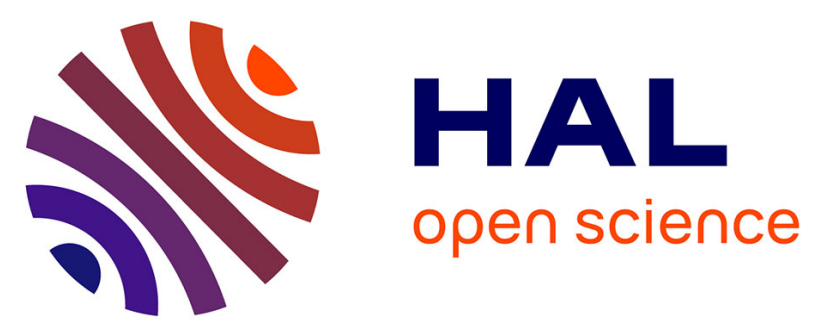

\title{
HgTe, the Most Tunable Colloidal Material: from the Strong Confinement Regime to $\mathrm{THz}$ Material
}

Clément Livache, Nicolas Goubet, Bertille Martinez, Eva Izquierdo, Charlie Gréboval, Sandrine Ithurria, Emmanuel Lhuillier

\section{To cite this version:}

Clément Livache, Nicolas Goubet, Bertille Martinez, Eva Izquierdo, Charlie Gréboval, et al.. HgTe, the Most Tunable Colloidal Material: from the Strong Confinement Regime to THz Material. MRS Advances, 2018, pp.1 - 9. 10.1557/adv.2018.409 . hal-01782488

\section{HAL Id: hal-01782488 \\ https://hal.science/hal-01782488}

Submitted on 25 Aug 2020

HAL is a multi-disciplinary open access archive for the deposit and dissemination of scientific research documents, whether they are published or not. The documents may come from teaching and research institutions in France or abroad, or from public or private research centers.
L'archive ouverte pluridisciplinaire HAL, est destinée au dépôt et à la diffusion de documents scientifiques de niveau recherche, publiés ou non, émanant des établissements d'enseignement et de recherche français ou étrangers, des laboratoires publics ou privés. 


\title{
HgTe, the Most Tunable Colloidal Material: from the Strong Confinement Regime to THz Material
}

\author{
Clément Livache ${ }^{1,2}$, Nicolas Goubet ${ }^{1,2}$, Bertille Martinez ${ }^{1,2}$, Eva Izquierdo ${ }^{2}$, Charlie \\ Greboval, ${ }^{1}$ Sandrine Ithurria ${ }^{2}$, Emmanuel Lhuillier ${ }^{1 *}$ \\ ${ }^{1}$ Sorbonne Université, UPMC Univ. Paris 06, CNRS-UMR 7588, Institut des NanoSciences de Paris, 4 \\ place Jussieu, 75005 Paris, France
}

${ }^{2}$ Laboratoire de Physique et d'Étude des Matériaux, ESPCI-Paris, PSL Research University, Sorbonne Université UPMC Univ Paris 06, CNRS, 10 rue Vauquelin 75005 Paris, France.

\begin{abstract}
HgTe nanocrystals are extremely interesting materials to obtain a highly tunable absorption spectrum in the infrared range. Here, we discuss the two extreme cases of strongly confined and barely confined HgTe nanocrystals. We discuss the synthesis and optoelectronic properties of HgTe 2D nanoplatelets where the confinement energy can be as large as $1.5 \mathrm{eV}$. This material presents enhanced (mostly narrower) light emitting properties compared to spherical nanocrystals emitting at the same wavelength. Moreover, absorption spectra, majority carriers and time response can be tuned by carefully choosing the surface chemistry and applying a well-chosen gate bias. HgTe can also be used to explore the effect of vanishing confinement and to obtain quasi bulk properties with tunable absorption in the THz, up to $150 \mu \mathrm{m}$.
\end{abstract}

\section{INTRODUCTION}

Since the introduction of the hot injection method [1] in the early 90's, the colloidal synthesis of nanocrystals has reached a high level of maturity. The synthesis of II-VI, III-V and IV-VI semiconductors can be achieved with a high level of monodispersity leading to discrete atom-like spectra even for ensemble solution measurements. Synthesis is no longer limited to core-only objects: complex heterostructures with various dimensionalities (0D spheres, 1D rods [2] and 2D platelets [3,4]) can be obtained in solution. Nanocrystals have reached a first mass market application with their introduction as the next generation of LCD display phosphors to build gamut-enhanced displays. More applications might come from optoelectronics, including solar cells, infrared sensitization of CMOS technology [5] and infrared sensors [6,7].

The infrared range of wavelengths is also of utmost interest for colloidal quantum dots (CQDs). Indeed, current technologies based on epitaxially-grown semiconductors remain often pricy and low-cost alternatives such as organic electronics are ineffective in the infrared range. Thus, CQDs appear as a new candidate in the field by combining (i) tunable optical absorption up to the $\mathrm{THz}$ range [8] (ii) stability of inorganic materials and (iii) ease of processing of colloidal based materials. 
In the near infrared, lead chalcogenides $(\mathrm{PbX})$ are by far the most investigated materials and they have reached a high success for solar cell design [9]. When longer wavelengths are necessary (from $5 \mu \mathrm{m}$ and beyond), $\mathrm{PbX}$ materials are limited by their bulk band gap, that is why narrower band gap materials have to be synthetized. Two strategies can be investigated: (i) the use of very narrow band gap semiconductors or semimetals where confinement is mildly introduced to obtain infrared interband transitions; or (ii) alternatively, wide band gap doped semiconductors [10,11] can present intraband or plasmonic features in the mid and far infrared. This is typically the case for self-doped chalcogenides materials (HgS, [12-14] $\left.\mathrm{HgSe},[15,16] \mathrm{Ag}_{2} \mathrm{Se}[17,18]\right)$, doped silicon [19-21] and doped oxides [22-25].

However, when it comes to optoelectronics, the control of transport properties is as critical as the tunability of the absorption spectrum. In this sense, $\mathrm{HgTe}$ is certainly the most mature material [26] under colloidal form. Over the past decades, many groups have reported about near [27-30], mid [31,32] and far [33,34] infrared photoconductive properties [35,36] of HgTe nanocrystal films [37]. In spite of this result, the material still suffers from limitations such as sensitivity to air, [38] tendency to sinter even under mild temperature annealing and presence of heavy metal. In this proceeding, we discuss two key material improvements relative to $\mathrm{HgTe}$ nanocrystals that have been obtained over the recent years by our group: the synthesis of $2 \mathrm{D} \mathrm{HgTe}$ nanoplatelets $[39,40]$ and the extension of the optical properties up to the THz range [41].

\section{Optical properties of HgTe nanocrystals}

Under bulk form, HgTe is a semimetal, see Figure 1, with an inverted band ordering $[42,43]$. It is generally reported in the literature that $\mathrm{HgTe}$ presents a negative band gap [44]. This negative band gap actually means that the band with a $\Gamma_{6}$ symmetry, which in many conventional semiconductors is the conduction band is actually located below the Fermi level. The bands which are the closest to the Fermi level and will actually drive the optical and transport properties of $\mathrm{HgTe}$ have a $\Gamma_{8}$ symmetry. The valence band is actually poorly dispersive, while the conduction band presents a relatively low effective mass which is responsible for the high mobility observed in bulk and epitaxially-grown $\mathrm{HgTe}$ films. For intrinsic HgTe, the Fermi level lies in the middle of these two bands (at zero energy on Figure 1). Once confinement is introduced (for sizes below $40 \mathrm{~nm}$ in the case of $\mathrm{HgTe}$ [45]), a (positive) energy transition appears between these two bands and its energy is potentially tunable down to 0 [46]. However, the large Bohr radius of $\mathrm{HgTe}$ makes that most materials reported so far remain in the strong confinement regime. 


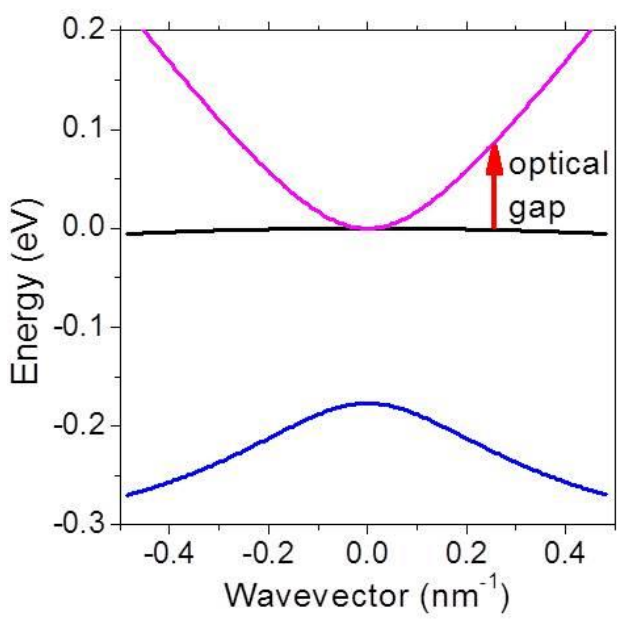

Figure 1 Simplified band structure of $\mathrm{HgTe}$.

In the following of this paper, we would like to discuss two extreme cases: the extremely confined regime where transition occurs in the near infrared and where confinement energy can be as high as $1.5 \mathrm{eV}$, and the other side where we obtain CQDs larger than $40 \mathrm{~nm}$ for which $\mathrm{THz}$ transitions are observed.

\section{Strongly confined HgTe nanoplatelets}

Among colloidal nanocrystals, II-VI nanoplatelets (NPLs) present very specific optical properties with certainly the narrowest excitonic features $[3,4]$. This narrow linewidth is the result of their very special growth mode which leads to roughness-free surface along the only confined direction. Growth of 2D NPLs has been reported with cadmium chalcogenides ( $\mathrm{CdS}, \mathrm{CdSe}$ and $\mathrm{CdTe}$ ) and their heterostructures, but nevertheless their spectral range is limited to visible wavelengths. Pushing their color tunability toward infrared range is of utmost interest to obtain infrared active colloidal materials with better defined excitonic features. Indeed, in the $800-1500 \mathrm{~nm}$ range, $\mathrm{PbS}$ nanocrystals are the most investigated materials but still present broad optical features resulting from homogeneous broadening and consequently leaving little room for synthesis optimization [47].

We recently report the synthesis of HgTe NPLs [39]. For the moment, direct synthesis to obtain such HgTe NPLs has been unsuccessful and our approach was rather to start from CdTe NPLs [48] and then perform a cation exchange step [49,50]. While introducing the $\mathrm{Hg}$ cations under complexed form with oleylamine, the cation exchange procedure keeps the 2D NPL aspect unaffected, as shown by electron microscopy, see Figure 2a. The final objects have a large lateral extension of several hundreds of nanometers while their thickness is only 1-2 nm. During this procedure, the initial excitonic feature of the CdTe NPLs (at $500 \mathrm{~nm}$ ) first disapears and then re-appears but is strongly redshifted, typically at $890 \mathrm{~nm}$, see Figure $2 \mathrm{~b}$. This corresponds to a confinement energy of $1.4 \mathrm{eV}$, which makes these HgTe NPLs among the most confined materials. As a result, the wavefunction partly leaks up to the ligands. Thus, by simply changing the capping ligands, it is possible to tune the exciton energy from $1.4 \mathrm{eV}$ for oleylamine-capped NPLs to $1.5 \mathrm{eV}$ with oleic acid-capped NPLs. 
HgTe nanocrystals are also interesting as near-IR light emitters [51-53]. In the case of NPLs, they present a narrow photoluminescence (PL) peak which full width at half maximum is $40 \mathrm{~nm}(60 \mathrm{meV})$. This is typically twice smaller than the value reported for $\mathrm{PbS}$ CQDs emitting at the same wavelength. The PL efficiency is around $10 \%$ while the PL lifetime is around $50 \mathrm{~ns}$, see Figure 2c.
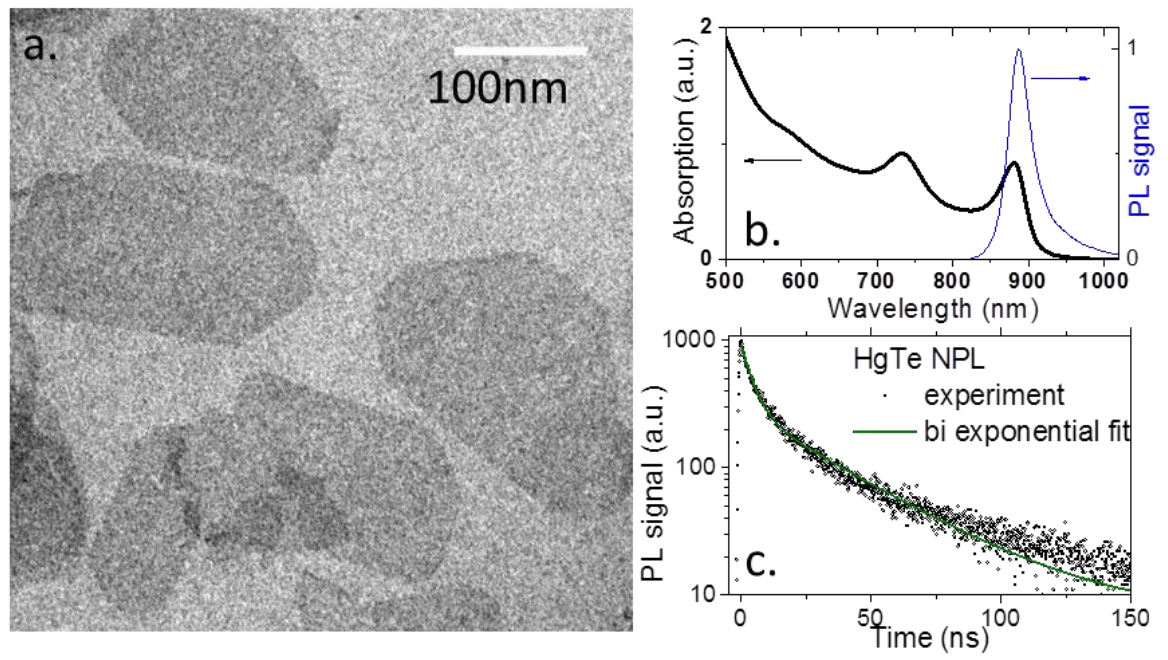

Figure 2a. Transmission Electron Microscopy (TEM) image of HgTe NPLs b. Absorption and photoluminescence spectrum of HgTe NPLs. c. Time resolved photoluminescence of HgTe NPLs.

We also have investigated the transport properties of these NPLs [40] in an electrolyte gating configuration [54] (see Figure 3a). It combines the advantages of air operability and being able to gate thick films, which is of utmost interest for the design of phototransistors [55]. We demonstrated that the nature of the majority carriers can be tuned with surface chemistry. Ethanedithiol (EDT) capped NPLs present p-type behavior (see Figure $3 b$ ), while $S^{2-}$ capped NPLs present a n-type behavior (see Figure 3c). Moreover, we notice that only the EDT-capped NPLs present a significant photoresponse, see Figure $3 \mathrm{~d}$. To understand these two results, we reconstructed the electronic spectra of the NPLs with these two surface chemistries by combining infrared spectroscopy and photoemission measurements made on the Tempo beamline of Soleil synchrotron, see Figure 3e. We can clearly see that EDT-capped HgTe NPLs almost behave as an intrinsic semiconductor, with the Fermi level lying close to the middle of the gap. This leads to a limited number of thermally activated carriers, and thus to a large photoresponse. On the other hand $\mathrm{S}^{2-}$ capped NPLs have their Fermi level almost resonant with the conduction band which favours thermal activation and injection of majority (electrons) carriers, at the price of a reduced photoresponse.

The ligand effect might appear in contradiction with recent reports $[12,56]$ on the effect of ligand exchange onto the electronic spectrum of mercury chalcogenides nanocrystals. Indeed it has been shown that for $\mathrm{HgSe}$ or $\mathrm{HgTe}$ quasi-spherical nanocrystals, $\mathrm{S}^{2-}$ ligands favour $\mathrm{p}$ type doping or at least reduce the $\mathrm{n}$ type character of the nanocrystals, while the opposite behaviour is observed for NPL. We attribute this discrepancy to the difference of main exposed facets. For quasi spherical nanocrystals, HRTEM reveals (111) facets as the most exhibited facets. On the other hand, in NPL the 
(001) facets are the most exposed facets. In this direction (001) only the cations are exposed, while for (111) plane the anion anions atoms are very close the surface. We thus believe that the difference of exposed surface atoms is responsible for the observed difference in the doping shift with surface chemistry.

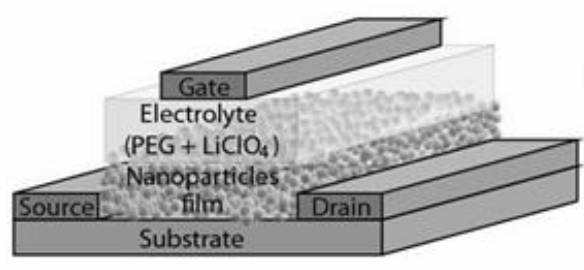

a.

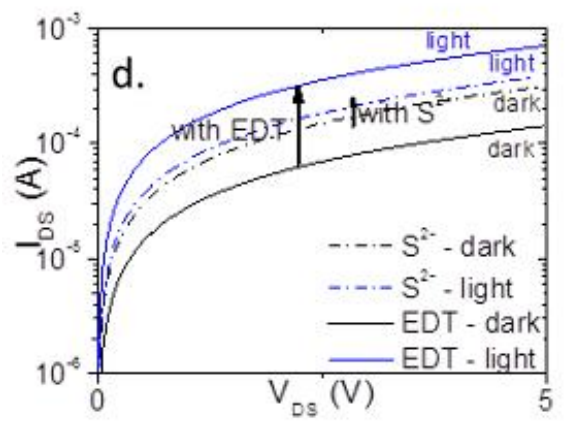

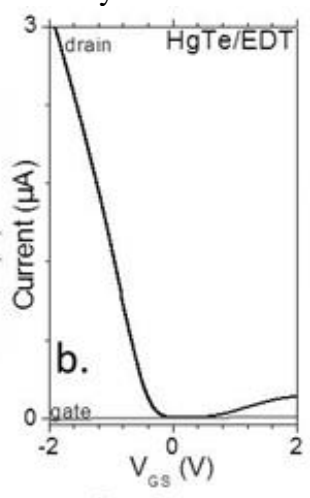
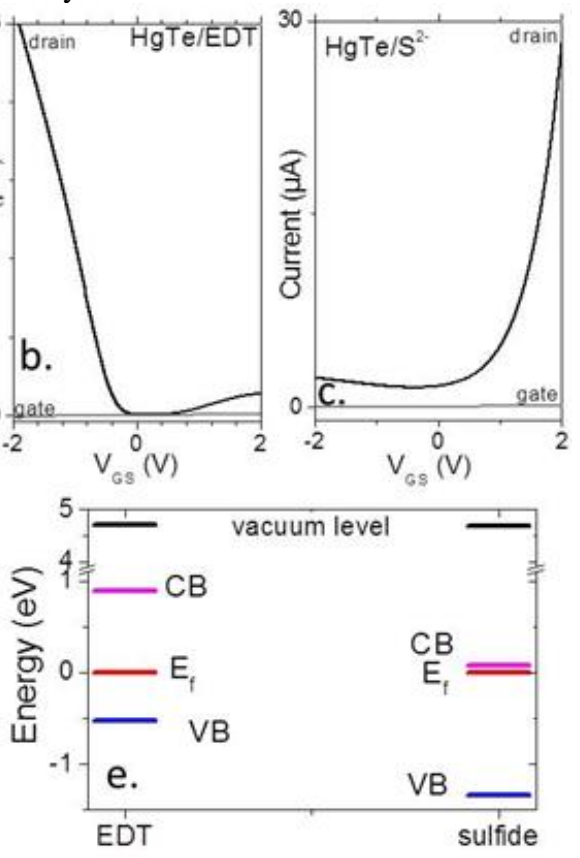

Figure 3a. Scheme of an electrolyte gated transistor. Transfer curve (drain and gate current as a function of the applied gate bias) for an EDT-capped HgTe NPL film (part b.) and for $\mathrm{S}^{2-}$-capped HgTe NPL film (part c.). d. I-V curve in dark condition and under illumination for HgTe NPL films capped with EDT and $\mathrm{S}^{2-}$. e. Reconstructed electronic spectrum for thin films of $\mathrm{HgTe}$ NPLs capped with EDT and $\mathrm{S}^{2-}$. CB: conduction band. VB: valence band. Ef: Fermi level.

The time response of EDT-capped HgTe NPL films is typically between $100 \mu \mathrm{s}$ and $1 \mathrm{~ms}$, see Figure 4a. The latter can actually be tuned with the applied gate bias. The gate can be used to empty the material of its majority carriers (holes in this case) and thus obtain a larger photocurrent modulation and a faster time response, see Figure 4b. 

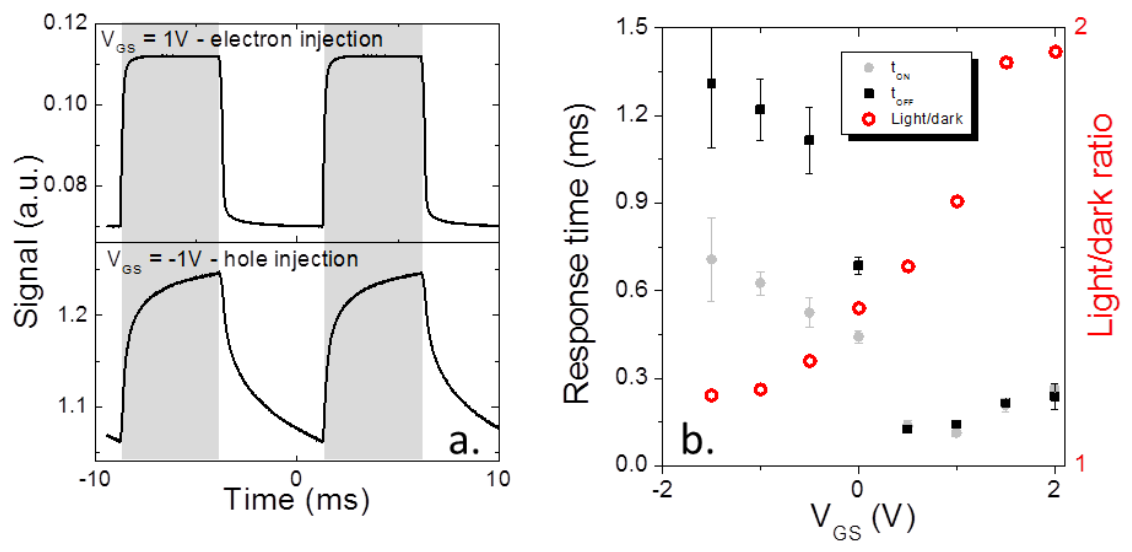

Figure 4 a. Current as a function of time for an EDT-capped HgTe NPL film under hole injection (negative gate bias) and under electron injection (positive gate bias) while the light is turned on and off. b. Time response and light to dark current modulation as a function of the applied gate bias for an EDT-capped HgTe NPL film.

\section{HgTe turning metallic: design strategy for THz nanocrystals}

Another synthetic challenge regarding HgTe relates to the synthesis of large particles to be able to push the absorption features really deeply in the infrared range [56]. Because of the large Bohr radius of $\mathrm{HgTe}(40 \mathrm{~nm})$, large nanoparticles have to be grown to recover the semimetallic behavior of the bulk material or to observe topological effect. We recently developed a new synthetic approach for the synthesis of HgTe CQDs [41]. Thanks to this procedure, the size of the HgTe CQDs can be tuned from $5 \mathrm{~nm}$ up to $1 \mu \mathrm{m}$, see Figure $5 \mathrm{a}$ and $\mathrm{b}$. As a result, the optical absorption can be tuned from the near-infrared up to the $\mathrm{THz}$ range. To grow such large $\mathrm{HgTe}$ nanoparticles it is critical to push the reaction temperature toward high temperature. Typically Keuleyan et al [57] synthesis was relying on growth temperature in the range from $60^{\circ} \mathrm{C}$ to $120^{\circ} \mathrm{C}$, here temperature as high as $300^{\circ} \mathrm{C}$ are used to obtain the largest size of nanoparticles.

As recently demonstrated by the Guyot-Sionnest group, n-type self-doping is observed in sufficiently large HgTe CQDs [34]. By increasing the size of CQDs above 100 $\mathrm{nm}$ [41], we have been able to obtain an absorption peak up to $60 \mu \mathrm{m}$ and up to $150 \mu \mathrm{m}$ for the cut-off wavelength. The $\mathrm{THz}$ feature actually overlaps with the absorption resulting from LO phonon which in the case of $\mathrm{HgTe}$ occurs at $125 \mathrm{~cm}^{-1}$, see Figure $5 \mathrm{c}$. This is, to our knowledge, the reddest colloidal material reported so far. 

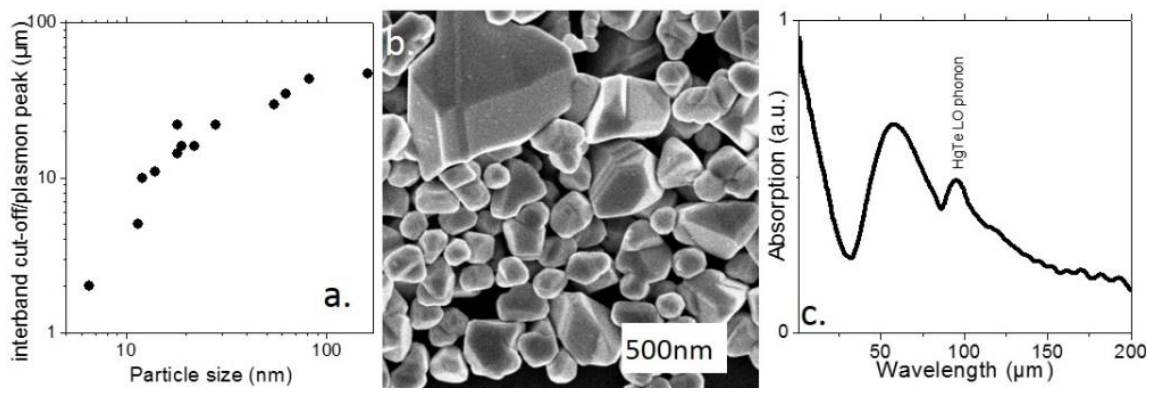

Figure $5 \mathrm{a}$. Energy of the reddest optical feature of $\mathrm{HgTe}$ nanocrystal as a function of the nanoparticle size. b. SEM image of large HgTe nanocrystals. c. Infrared spectrum of large HgTe nanocrystals with absorption in the THz range.

\section{CONCLUSIONS}

HgTe nanocrystals are an interesting platform to explore the effect of confinement from the strongly confined regime to the bulk material properties. 2D NPLs of HgTe are among the most confined colloidal materials. They present enhanced emission properties in the near-IR range resulting from their 2D growth mechanism. In addition, they present fast photoconduction properties. Moreover, by carefully tuning the surface chemistry and applying a gate voltage, majority carrier nature and dynamics can be finely tuned. At the complete opposite range, we demonstrate the synthesis of HgTe nanocrystals where the confinement is vanishing and with absorption in the $\mathrm{THz}$ range, which are the reddest colloidal materials currently reported.

\section{ACKNOWLEDEGMENTS}

We acknowledge the use of cleanroom facilities from the consortium "Salles Blanches Paris Centre -SBPC". This work has been supported by the Region Ile-de-France in the framework of DIM Nano-K via the grant dopQD. This work was supported by French state funds managed by the ANR within the Investissements d'Avenir program under reference ANR-11-IDEX-0004-02, and more specifically within the framework of the Cluster of Excellence MATISSE, as well as by grant Nanodose and H2DH. EL is thankful for the support of the ERC starting grant (project: blackQD - grant 756225).

\section{References}

1. C. B. Murray, D. J. Norris, and M. G. Bawendi, J. Am. Chem. Soc. 115, 8706-8715 (1993).

2. X. Peng, L. Manna, W. Yang, J. Wickham, E. Scher, A. Kadavanich, and A. P. Alivisatos, Nature 404, 59-61 (2000).

3. M. Nasilowski, B. Mahler, E. Lhuillier, S. Ithurria, and B. Dubertret, Chem. Rev. 116, 10934-10982 (2016).

4. E. Lhuillier, S. Pedetti, S. Ithurria, B. Nadal, H. Heuclin, and B. Dubertret, Acc. Chem. Res. 48, 22-30 (2015).

5. V. Adinolfi and E. H. Sargent, Nature 542, 324-327 (2017). 
6. M. V. Kovalenko, E. Kaufmann, D. Pachinger, J. Roither, M. Huber, J. Stangl, G. Hesser, F. Schäffler, and W. Heiss, J. Am. Chem. Soc. 128, 3516-3517 (2006).

7. S. Keuleyan, E. Lhuillier, V. Brajuskovic, and P. Guyot-Sionnest, Nat. Photonics 5, 489493 (2011).

8. E. Lhuillier, M. Scarafagio, P. Hease, B. Nadal, H. Aubin, X. Z. Xu, N. Lequeux, G. Patriarche, S. Ithurria, and B. Dubertret, Nano Lett. 16, 1282-1286 (2016).

9. Konstantatos and Sargent, Collidal Quantum Dot Optoelectronics and Photovoltaics (Cambridge University Press, 2013).

10. C. Wang, M. Shim, and P. Guyot-Sionnest, Science 291, 2390-2392 (2001).

11. D. J. Norris, A. L. Efros, and S. C. Erwin, Science 319, 1776-1779 (2008).

12. K. S. Jeong, Z. Deng, S. Keuleyan, H. Liu, and P. Guyot-Sionnest, J. Phys. Chem. Lett. 5, 1139-1143 (2014).

13. B. Yoon, J. Jeong, and K. S. Jeong, J. Phys. Chem. C 120, 22062-22068 (2016).

14. G. Shen and P. Guyot-Sionnest, J. Phys. Chem. C 120, 11744-11753 (2016).

15. X. Tang, G. fu Wu, and K. W. C. Lai, J. Mater. Chem. C 5, 362-369 (2016).

16. Z. Deng, K. S. Jeong, and P. Guyot-Sionnest, ACS Nano 8, 11707-11714 (2014).

17. A. Sahu, L. Qi, M. S. Kang, D. Deng, and D. J. Norris, J. Am. Chem. Soc. 133, 6509-6512 (2011).

18. A. Sahu, A. Khare, D. D. Deng, and D. J. Norris, Chem. Commun. 48, 5458-5460 (2012).

19. T. Chen, K. V. Reich, N. J. Kramer, H. Fu, U. R. Kortshagen, and B. I. Shklovskii, Nat. Mater. 15, 299-303 (2016).

20. H. Zhang, R. Zhang, K. S. Schramke, N. M. Bedford, K. Hunter, U. R. Kortshagen, and P. Nordlander, ACS Photonics 4, 963-970 (2017).

21. R. Gresback, N. J. Kramer, Y. Ding, T. Chen, U. R. Kortshagen, and T. Nozaki, ACS Nano 8, 5650-5656 (2014).

22. R. Buonsanti, A. Llordes, S. Aloni, B. A. Helms, and D. J. Milliron, Nano Lett. 11, 47064710 (2011).

23. C. Delerue, Nano Lett. 17, 7599-7605 (2017).

24. E. Della Gaspera, M. Bersani, M. Cittadini, M. Guglielmi, D. Pagani, R. Noriega, S. Mehra, A. Salleo, and A. Martucci, J. Am. Chem. Soc. 135, 3439-3448 (2013).

25. B. Tandon, A. Yadav, D. Khurana, P. Reddy, P. K. Santra, and A. Nag, Chem. Mater. 29, 9360-9368 (2017).

26. E. Lhuillier and P. Guyot-Sionnest, IEEE J. Sel. Top. Quantum Electron. 23, 6000208 (2017).

27. S. Hyuk Im, H. Kim, S. Woo Kim, S.-W. Kim, and S. Il Seok, Nanoscale 4, 1581-1584 (2012).

28. M. Chen, L. Shao, S. V. Kershaw, H. Yu, J. Wang, A. L. Rogach, and N. Zhao, ACS Nano 8, 8208-8216 (2014).

29. H. Seong, K. Cho, and S. Kim, Semicond. Sci. Technol. 23, 075011 (2008).

30. M. Chen, H. Yu, S. V. Kershaw, H. Xu, S. Gupta, F. Hetsch, A. L. Rogach, and N. Zhao, Adv. Funct. Mater. 24, 53-59 (2013).

31. E. Lhuillier, S. Keuleyan, P. Rekemeyer, and P. Guyot-Sionnest, J. Appl. Phys. 110, 033110 (2011).

32. P. Guyot-Sionnest and J. A. Roberts, Appl. Phys. Lett. 107, 253104 (2015).

33. S. E. Keuleyan, P. Guyot-Sionnest, C. Delerue, and G. Allan, ACS Nano 8, 8676-8682 (2014).

34. G. Shen, M. Chen, and P. Guyot-Sionnest, J. Phys. Chem. Lett. 8, 2224-2228 (2017).

35. E. Lhuillier, S. Keuleyan, H. Liu, and P. Guyot-Sionnest, Chem. Mater. 25, 1272-1282 (2013).

36. Y. Yifat, M. Ackerman, and P. Guyot-Sionnest, Appl. Phys. Lett. 110, 041106 (2017).

37. B. Martinez, C. Livache, N. Goubet, A. Jagtap, H. Cruguel, A. Ouerghi, E. Lacaze, M. G. Silly, and E. Lhuillier, J. Phys. Chem. C 122, 859-865 (2018).

38. E. Lhuillier, S. Keuleyan, P. Zolotavin, and P. Guyot-Sionnest, Adv. Mater. 25, 137-141 (2013).

39. E. Izquierdo, A. Robin, S. Keuleyan, N. Lequeux, E. Lhuillier, and S. Ithurria, J. Am. Chem. Soc. 138, 10496-10501 (2016).

40. C. Livache, E. Izquierdo, B. Martinez, M. Dufour, D. Pierucci, S. Keuleyan, H. Cruguel, L. Becerra, J. L. Fave, H. Aubin, A. Ouerghi, E. Lacaze, M. G. Silly, B. Dubertret, S. Ithurria, and E. Lhuillier, Nano Lett. 17, 4067-4074 (2017). 
41. N. Goubet, A. Jagtap, C. Livache, B. Martinez, H. Portalès, X. Z. Xu, R. P. S. M. Lobo, B. Dubertret, and E. Lhuillier, J. Am. Chem. Soc. 140, 5033-5036 (2018).

42. G. Allan and C. Delerue, Phys. Rev. B 86, 165437 (2012).

43. G. Nimtz, B. Schlicht, and R. Dornhaus, Narrow-Gap Semiconductors (Springer, 1983).

44. P. Man and D. S. Pan, Phys. Rev. B 44, 8745-8758 (1991).

45. V. Rinnerbauer, K. Hingerl, M. Kovalenko, and W. Heiss, Appl. Phys. Lett. 89, 193114 (2006).

46. E. Lhuillier, S. Keuleyan, and P. Guyot-Sionnest, Nanotechnology 23, 175705 (2012).

47. M. P. Hendricks, M. P. Campos, G. T. Cleveland, I. J.-L. Plante, and J. S. Owen, Science 348, 1226-1230 (2015).

48. S. Pedetti, B. Nadal, E. Lhuillier, B. Mahler, C. Bouet, B. Abécassis, X. Xu, and B. Dubertret, Chem. Mater. 25, 2455-2462 (2013).

49. L. De Trizio and L. Manna, Chem. Rev. 116, 10852-10887 (2016).

50. B. J. Beberwyck, Y. Surendranath, and A. P. Alivisatos, J. Phys. Chem. C 117, 1975919770 (2013).

51. A. L. Rogach, S. V. Kershaw, M. Burt, M. T. Harrison, A. Kornowski, A. Eychmüller, and H. Weller, Adv. Mater. 11, 552-555 (1999).

52. P. Geiregat, A. J. Houtepen, L. K. Sagar, I. Infante, F. Zapata, V. Grigel, G. Allan, C. Delerue, D. Van Thourhout, and Z. Hens, Nat. Mater. 17, 35-42 (2018).

53. A. L. Rogach, A. Eychmüller, S. G. Hickey, and S. V. Kershaw, Small 3, 536-557 (2007).

54. E. Lhuillier, S. Ithurria, A. Descamps-Mandine, T. Douillard, R. Castaing, X. Z. Xu, P.-L. Taberna, P. Simon, H. Aubin, and B. Dubertret, J. Phys. Chem. C 119, 21795-21799 (2015).

55. E. Lhuillier, A. Robin, S. Ithurria, H. Aubin, and B. Dubertret, Nano Lett. 14, 2715-2719 (2014).

56. B. Martinez, C. Livache, L. D. Notemgnou Mouafo, N. Goubet, S. Keuleyan, H. Cruguel, S. Ithurria, H. Aubin, A. Ouerghi, B. Doudin, E. Lacaze, B. Dubertret, M. G. Silly, R. P. S. M. Lobo, J.-F. Dayen, and E. Lhuillier, ACS Appl. Mater. Interfaces 9, 36173-36180 (2017).

57. S. Keuleyan, E. Lhuillier, and P. Guyot-Sionnest, J. Am. Chem. Soc. 133, 16422-16424 (2011). 